\title{
Primary Malignant Melanoma of Male Urethra with Fistula
} Formation

\begin{tabular}{|l|l|l|}
\hline T. & Tatsuo & Morita $^{\mathrm{a}}$ \\
\hline H. & Hiroshi & Suzuki $^{\mathrm{a}}$ \\
\hline K. & Kentaro & Goto $^{\mathrm{a}}$ \\
\hline N. & Norio & Hirota $^{\mathrm{b}}$ \\
\hline A. & Akihiko & Tokue $^{\mathrm{a}}$ \\
\hline
\end{tabular}

Departments of ${ }^{\mathrm{a}}$ Urology and ${ }^{\mathrm{b}}$ Pathology, Jichi Medical School, Tochigi, Japan

\section{Key Words}

Malignant melanoma

Male urethra

Urethral fistula

\section{Abstract}

We report a case of primary malignant melanoma of the male urethra in whom fistula formation was observed in the fossa navicularis.

Tatsuo Morita, MD, Department of Urology, Jichi Medical School, Minamikawachi-machi, Kawachi-gun Tochigi 32904 (Japan)

Introduction

Cutaneous malignant melanoma is not an uncommon disease but primary malignant melanoma of the genitourinary tract is quite rare. Especially, primary malignant melanoma of the male urethra is so rare that only 23 cases have, thus far, been reported in the literature [1-4]. We report a case of primary malignant melanoma of the male urethra with fistula formation.

Case Report

A 75-year-old Japanese man was admitted to Jichi Medical School Hospital in March 1986 with complaints of dysuria and a penile mass that had been present for 2 years. On physical examination, a palpable urethral mass was noted in the distal urethra. The urethral fistula was observed in the fossa navicularis, and a blackened tumor, whitish in part, was protruding outside of the meatus and the urethral fistula (fig. 1).

Fig. 1. Macroscopic appearance of the urethral melanoma. The blackened tumor, whitish in part, was protruding outside of the meatus and the urethral fistula which was located in the fossa navicularis.

Fig. 2. Microscopic appearance of the urethral melanoma. The tumor consisted of round or spheroidal large cells with large hyperchromatic nuclei. There were many brown pigments in intra-cellular and extracellular spaces. HE. × 200 .

primary Malignant Melanoma of Male Urethra with Fistula Formation 115

The inguinal lymph nodes were not enlarged on palpation. There was no evidence of nevi or other cutaneous pigmented lesions. Urinary cytologic examination demonstrated atypical 
pigmented cells suggestive of malignant melanoma. Hematologic and blood chemistry data were normal. Chest X-ray film, excretory urogram (IVP), lymphangiogram, CT and bone scintigram were negative. Diagnosis of primary malignant melanoma of the distal urethra was made. Since there was no evidence of metastasis, the patient underwent penile amputation and bilateral inguinal lymph node dissection on April 22, 1986. Simultaneous cystourethroscopy revealed no other lesions in proximal urethra and bladder. Gross examination of the specimen revealed a blackened tumor measuring $6 \times 5 \times 25 \mathrm{~mm}$ extending from the fossa navicularis to the meatus. The tumor protruded outside of the meatus and the urethral fistula in the fossa navicularis, but there was no involvement of the skin of the glans penis. Histological examination of the tumor revealed malignant melanoma of the urethra with invasion into the corpus cavernosum. The tumor consisted of closely spaced, round or spheroidal large cells with large hyperchromatic nuclei (fig. 2). Many brown pigments stained black with the Fontana-Masson technique were seen in the cytoplasm of some tumor cells and extracellular space. Meta-static melanoma cells were found in one of the lymph nodes dissected. The postoperative course was uneventful. Based on pathological findings, the patient subsequently was treated with adjuvant chemotherapy with DTIC, ACNU and VCR. However, the patient died of systemic metastatic disease 18 months after the operation and autopsy was not performed.

Comment

The most common malignant neoplasms in the urethra are squamous cell carcinoma and transitional cell carcinoma, and melanoma of the male urethra is an even less common entity. A review of the literature revealed 24 cases of primary malignant melanoma of the male urethra including the present case [1-4]. The urethral melanomas reported in the literature have occurred at various levels of the urethra. The fossa navicularis (52\%) was the most common occurrence site, and the other reported sites included bulbus urethra (15\%), prostatic urethra (15\%), pendulous urethra (10\%) and meatus (8\%). Symptoms at the time of initial presentation were various, depending on the occurrence site, size and stage

of the tumor. They consisted of urethral mass, dysuria, hematuria, bloody penile discharge and split urinary stream. In the present case, a urethral fistula was observed in the fossa navicularis. This fistula formation is quite rare and may be attributed not only to the aggressive biological properties of melanoma cells such as highly invasive growth [5] but also delay in presentation. Concerning the latter, Weiss et al. [1] reported that the mean interval from onset of symptoms to presentation was 24.2 months in patients with urethral melanoma. In fact, the patient reported here was admitted to the hospital 2 years after the recognition of symptoms. This great lag period seems to lead to advance of the disease, especially formation of the urethral fistula.

References

Weiss J, Elder D, Hamilton R: Melanoma of the male urethra: Surgical approach and pathological analysis. J Urol 1982; 128: 382-385.

Kokotas NS, Kallis EG, Fokitis PJ: Primary malignant melanoma of male urethra. Urology 1981;153:392-394.

Begun FP, Grossman HB, Diokno AC, Sogani PC: Malignant melanoma of the penis and male urethra. J Urol 1984; 132:123-125.

Sanders TJ, Venable DD, Sanusi ID: Primary malignant melanoma of the urethra in a black man: A case report. J Urol 1986; 135:1012-1014.

Hujanen TT

Thorgeirsson UP

Rao CN 
Liotta LA: Laminin increases the release of type IV collagenase from malignant cells. J Biol Chem 1986;261:1883-1889.
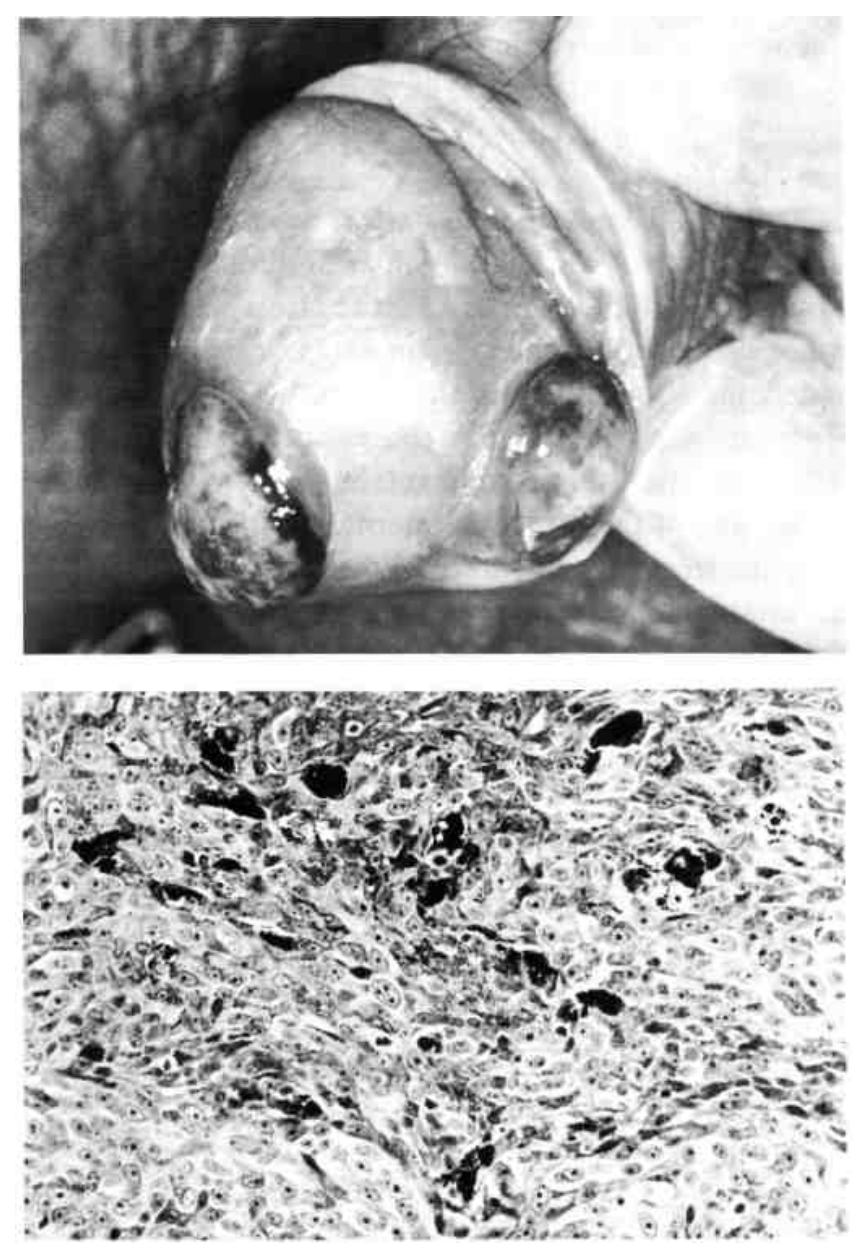